Europe's Journal of Psychology 4/2009, pp. 13-19

www.ejop.org

\title{
Health Research on the Current Agenda of School Psychology: Interview with Prof. Bonnie Nastasi
}

\section{By Vlad Glăveanu \\ EJOP Editor}

Health research, principally with a focus on student mental health and well-being, is a rising field in school psychology. The importance of the topic needs no explanation and yet, at the same time, there are both possibilities and challenges school psychologists face when engaging with this particular type of research, especially when studying cultural differences. One of the leading experts in this emerging field is Prof. Bonnie Nastasi who kindly accepted to answer our questions about the present state and the future of mental health research within school psychology.

Bonnie Kaul Nastasi, Ph.D., is an Associate Professor in the Department of Psychology in the School of Science and Engineering at Tulane University, New Orleans, Lovisiana, USA. Professor Nastasi received her Ph.D. in School Psychology in 1986 from Kent State University, Ohio, USA. Professor Nastasi's research focuses on the development and evaluation of culturally appropriate assessment and intervention approaches designed to promote mental health and reduce health risks such as STIS/HIV, both within the US and internationally. She has worked in Sri Lanka since 1995 on development of school-based programs to promote children's and adolescent's psychological well-being, and is currently directing a multi-country study to examine the psychological well-being of children and adolescents, with research partners in 12 countries. She has worked in India for the past 7 years as one of the principal investigators of an interdisciplinary public health research project, focused on prevention of HIV/STIs among married men and women living in the slums of Mumbai. Professor Nastasi has published extensively, with over 60 journal articles and chapters, and three books. Her most recent book, School-based Mental Health Services: Creating Comprehensive and Culturally Specific Programs, was published by the American Psychological Association (APA) in 2004.

Professor Nastasi is currently the President-elect for Division 16 (School Psychology) of the American Psychological Association. 
EJOP: Dr. Nastasi, your extensive work over the past decades constitutes a significant contribution to both health psychology and school psychology and emphasizes the links between the two, reminding us of the importance of health issues, especially mental health, for school psychologists. How would you comment on the evolution and current state of health research and risk prevention within school psychology?

Bonnie Nastasi: Health research and risk prevention in school psychology is an emerging field, but of course builds on a large body of existing research within medical and public health fields. At least within the academic community, school psychology researchers are increasingly cognizant of the importance of health and mental health in understanding and fostering children's functioning in schools. I see the field as moving toward a broader focus consistent with emerging integrated theories in psychology that emphasize the links among biological, psychological, social and cultural factors (i.e., biopsychosocial) and the importance of the ecology to child development and functioning. Moreover, the field of psychology has been influenced by the 'positive psychology' movement which can serve to broaden the traditional deficit orientation to one focused on risk prevention and promotion of well-being. One of the other influences at an applied level is the increasing concern for impact of disasters and crises that children experience, and the need for enhancing children's resilience. Another influence, particularly within the US, is the increasing concern about nutrition and obesity and the need for more prevention oriented health programming. Of course research that involves school psychology is critical to addressing such issues. I expect that such concerns will continue to influence our research in school psychology, so that we can contribute to efforts to understand the social and behavioral aspects of health and illness and to develop programming to foster healthy development.

EJOP: How is the concern for the mental health and well-being of students reflected today in schools and the work of school psychologists? What is done and what should be done in the future, in the US and worldwide?

Bonnie Nastasi: There is growing interest and focus at the practice level of school psychology regarding physical and mental health, although this is still an emerging area in the field. I attribute that to three factors. One is the gap between theoryresearch and practice, so that advances in theory and research generated in universities are not necessarily influencing practice in schools. Second, particularly in the US, is the strong focus on academic achievement in the education community, to the detriment of health and mental health. Third is the traditional deficit orientation in psychology and restricted focus on treatment for those with identifiable (diagnosable) disorders, to the detriment of risk prevention and health 
promotion efforts. Despite the challenges, there is an increasing recognition of the links among health, mental health, and academic achievement and need for more integrated theories such as biopsychosocial, which I expect will lead to increased efforts to better understand these links and to promote the overall well-being of students in schools. Unfortunately, mental health efforts in schools are insufficient to meet the demand for services due to the insufficiency of funding, providers, and training programs. This of course limits the attention to prevention and health promotion, as the restricted services must be provided to those most in need. With few exceptions, this is a problem in all countries. Where mental health services exist, there is frequently lack of coordination among different providers within the school and between school and community.

The solution is complex. Of course, more mental health professionals would be helpful, but this also requires increased funding and has policy implications at national, regional, and local levels. This also has implications for training programs, especially in countries where school psychology is limited. At a practice level, expanding the focus of school psychological services becomes critical, which means broader theoretical model as I noted in my earlier response and ensuring that school psychologists are equipped with skills in consultation, prevention and mental health promotion, program development and evaluation research, and systemic change. One of the challenges for training programs is that many school psychologists still operate in a traditional manner, restricting relevant practicum experiences. Overcoming this challenge may require that faculty work directly with students in local schools to help institute changes in the local system. More fully addressing the mental health needs of students, particularly with a focus on promoting well-being, will require that school psychologists collectively and individually become effective leaders and advocates for change at global, national and local levels.

EJOP: You talked at length in your published work about school-based mental health services, at the same time highlighting the necessity of developing culture-specific programs for both prevention and intervention. Which are the steps to be to taken in order to develop such programs? What challenges do we face on the way?

Bonnie Nastasi: Developing culture-specific programs requires that we conduct formative research to examine individual, cultural, and contextual factors that are related to children's psychological well-being, prior to selection, adapting or developing appropriate school-based mental health programs. With colleagues, Rachel Moore and Kris Varjas, we have developed a model for program 
development, the Participatory Culture-Specific Intervention Model (PCSIM'), which involves a series of steps and procedures necessary for system entry, forming partnerships, learning the local culture, conducting formative research that informs a local model of practice, and conducting both formative and summative evaluation to inform contextual adaptations, examine program success, and promote sustainability. Of course, a critical component of the model is partnership / participation, which requires identifying and involving representatives of key stakeholders (those with vested interests or resources) in the PCSIM process. The model is designed to promote acceptability and cultural validity of interventions, which in turn can help to ensure program integrity and effectiveness.

A primary challenge is time. The process is systematic and multi-phase. However, the time is well invested as you are more likely to develop a program that meets the specific needs of the context (school/community), and stakeholders are likely to be more invested in the project and are more likely to sustain the efforts. Of course, doing this work requires that school psychologists have relevant skills in consultation, facilitating group process, and program development and evaluation research. Using an approach like PCSIM is not a quick fix, thus school psychologists need to be prepared to help schools address the immediate or short-term problems while developing long-term programming. Ultimately, the proof lies in documenting program effectiveness in the specific setting.

EJOP: This leads us to the broad topic of culture and the social environment in general and cultural differences in particular. This interest became more and more prominent in mental health studies. How is the 'ecological approach' reflected today in school psychology?

Bonnie Nastasi: The ecological approach does seem to be well accepted in the field of school psychology as a model for research and practice. Within the US, I see this as the current predominant model, although other theoretical approaches are still prevalent and may influence specific aspects of practice. The ecological approach also is consistent with the movement toward integrated theoretical models (e.g., biopsychsocial) at least within American psychology, which attempt to bring together existing theories in order to address the complexity of human behavior and change. The ecological approach is reflected in the recognition of the critical role of culture and social environment both in research and practice, the efforts to involve parents and families in more meaningful ways in children's education and mental health promotion, and the recognition of the importance of

1 Nastasi, B.K., Moore, R. B., \& Varjas, K. M. (2004). School-based mental health services: Creating comprehensive and culturally specific programs. Washington, DC: American Psychological Association. 
community. I view the ecological approach in school psychology as an indicator of the more general recognition of the importance of interdisciplinary research in fields related to human functioning, particularly education and public health. Certainly, work in risk prevention and health promotion in general has become increasingly interdisciplinary in both research and practice arenas. I see school psychology as moving in that direction, although that does require some reframing by the thinkers in our field. The challenge is to move outside of our specific theoretical and topicspecific interests and be willing to work with researchers and practitioners who are likely to challenge our theoretical perspectives.

EJOP: The 'ecological approach' is also associated with research designs that favor qualitative methods or mixed-methods. You have often employed qualitative methods in the study of socio-cultural settings. What would be the advantages and the challenges of using such an approach?

Bonnie Nastasi: At this point, I consider myself to be a mixed methodologist. Especially when conducting research with intervention and change as the ultimate goals, it is hard for me to conceive of research without a mixed method approach. In brief, qualitative research has much to contribute to more traditional quantitative designs that have dominated psychology, and the combination of qualitative and quantitative methods provides optimal methods for developing culturally and contextually relevant understandings and practices. For example, mixed methods research is critical to the PCSIM I described earlier. In particular, qualitative methods can help us to understand cultural meaning and variations in constructs across individuals and groups and to expand our theoretical understandings beyond the existing theories which are primarily based on Western notions of human behavior. Qualitative methods also can help us to understand the contextual variables relevant to human behavior, consistent with an ecological model. Finally, qualitative methods can help us to document and understand the process of intervention implementation in order to both explain outcomes and inform replications or adaptations. Mixed methods combine these benefits with those of more traditional quantitative methods, which help us to develop aggregate depictions of behavior, predict behavior, and generalize to populations. The primary challenge of qualitative and mixed methods is the lack of training among psychologists and particularly school psychologists. Conducting qualitative and mixed methods research requires specific skills in data collection, analysis, and inference in order to ensure appropriate quality of findings. The other challenge is the time consuming nature of such work. Based on my own experiences, the additional resources are well justified by the benefits that accrue. 
EJOP: Much of your research has taken place in Sri-Lanka and other Asian countries. What lessons have you learned from working in a different cultural setting? How easy or difficult it is to "enter" a different culture and to "understand" it, avoiding Westerncentric interpretations?

Bonnie Nastasi: Probably the most important lesson from my work in Asia (particularly Sri Lanka and India) is to question my assumed understanding of cultures within my own country and community. I think we make assumptions about others based on our own worldview, and it is easy to impose our own views and misinterpret others based on our own experiences. I have come to appreciate both the diversity and the commonality of human experience from my work in other cultures. My work in Asia has increased my appreciation of an ecological perspective-to understand the importance of culture and context as it influences human behavior and cognition. In one-to-one interactions, this appreciation is reflected in an effort to truly understand the perspective of others, to learn about their life experiences historically and currently, to be more cautious in my judgments of others, and to learn from others in order to enhance my own understanding and well-being. This work has definitely made me more humble! The humility is in part a function of the challenges in entering and living within a culture that is unfamiliar to me. With practice, the process of entry can become easier but involves time and patience and a willingness to experience failures and learn from others. It is, of course, critical to be aware of one's ethnocentric perspective and to monitor that in interacting with others. Most importantly, one must be willing to broaden that perspective to encompass the experiences and views relevant to other individuals and cultures. This takes motivation, effort and practice, but for me has been life enhancing.

EJOP: YOU are at the moment coordinating an international research project concerned with developing culture-specific conceptualizations and interventions for increasing students' well-being. Can you tell us a bit more about this project, its aims and methods?

Bonnie Nastasi: The project, initiated as a joint venture by International School Psychology Association (ISPA) and the Society for the Study of School Psychology (SSSP; US-based), is designed to explore the culture-specific conceptualizations of psychological well-being (i.e., mental health) and mechanisms for promoting wellbeing from the perspectives of school-age children and adolescents, their parents and teachers, school administrators, and school physical or mental health providers. Each country-based research partner collects data within their own community/country context. The goal is to analyze the data to identify common and unique aspects of psychological well-being based on cultural conceptions. We 
expect to have participation from about 12 countries. Currently, the project is in data collection and analysis phases with some variations across countries. The ultimate goal is to develop culture-specific interventions to promote psychological well-being in participating contexts. Anyone interested in the project can contact me at bnastasi@tulane.edu.

EJOP: Finally, what would be your advice for our readers who are now pursuing a career in school psychology and are interested in enhancing the mental health and therefore the well-being of students? What can be done at the micro-level of each school, of each classroom?

Bonnie Nastasi: First and foremost, study school psychology from a broad theoretical and methodological perspective, so that you are prepared to work across disciplines, understand the complexity of human behavior from an integrated theoretical perspective, and make use of mixed methods research to inform your practice. Perhaps most importantly, in the age of 'evidence-based practice', it is important that you critically examine the 'evidence' underlying the practices. Do not assume that programs identified by others as effective in some context or with a certain population are necessarily going to be effective, or even appropriate, for your specific site. Be a critical consumer with regard to the cultural and contextual relevance of the available evidence, and learn to combine the best available evidence with appropriate strategies for adapting those to ensure effectiveness in your target context. At the classroom level, school psychologists can serve as consultants or collaborators with teachers in instituting interventions to promote psychological well-being of all children, and to identify and provide appropriate interventions for those who need more intensive services. 